\title{
Lentiviral-Mediated shRNA Silencing of PDE4D Gene Inhibits Platelet-Derived Growth Factor-Induced Proliferation and Migration of Rat Aortic Smooth Muscle Cells
}

\author{
Lin Liu, ${ }^{1}$ Xiaowei Xu, ${ }^{2}$ Jiejie $\mathrm{Li}^{2}{ }^{2} \mathrm{Xia} \mathrm{Li}^{2}$ and Wenli Sheng ${ }^{2}$ \\ ${ }^{1}$ Department of Neurology, Sun Yat-Sen University Tungwah Hospital, Dongguan, China \\ ${ }^{2}$ Stroke Center Department of Neurology, First Affiliated Hospital, Sun Yat-Sen University, Guangzhou, China
}

Correspondence should be addressed to Wenli Sheng, wenlisheng@hotmail.com

Received 30 November 2010; Accepted 3 March 2011

Academic Editor: Daniel Woo

Copyright $\odot 2011$ Lin Liu et al. This is an open access article distributed under the Creative Commons Attribution License, which permits unrestricted use, distribution, and reproduction in any medium, provided the original work is properly cited.

\begin{abstract}
Phosphodiesterase 4D (PDE4D) is a member of the large superfamily of phosphodiesterases. PDE4D polymorphisms have been found to associate with ischemic stroke. Proliferation and migration of vascular smooth muscle cells (VSMCs) play a critical role in the pathogenesis of atherosclerosis. In this study, infection of VSMCs with lentivrius particles carrying shRNA direct against PDE4D significantly inhibited platelet-derived growth factor-induced VSMC proliferation and migration, and the inhibitory effects were not associated with global intracellular cAMP level. Our results implicate that PDE4D has an important role in VSMC proliferation and migration which may explain its genetic susceptibility to ischemic stroke.
\end{abstract}

\section{Introduction}

Stroke, a complex disease, remains the third common cause of death behind heart disease and cancer and is the leading cause of disability in developed countries as well as in China. Ischemic stroke accounts for up to $80 \%$ of all strokes. Both environmental factors and genetic variants contribute to stroke. Although several genetic defects have been identified in rare forms of stroke, the genetic causes of common forms of ischemic stroke remain elusive. Recently, PDE4D has been identified as the first gene linked to common forms of stroke [1]. However, subsequent studies have produced inconsistent results for the association [2-9]. Due to the variable results, several meta-analyses on the gene have been accomplished, which also drew conflicting conclusions [10-13]. Racial differences might be responsible for the inconsistent results. Therefore, experimental studies on function of PDE4D will help to unveil the puzzle.

PDE4D belongs to a large superfamily of PDEs which are the only hydrolytic enzymes of cAMP and key signal transduction molecules in multiple cell types, including vascular smooth muscle cells. Eleven families (PDE1-PDE11) have been identified in the large superfamily of PDEs [14]. There are four major families found in VSMCs: PDE1, PDE5, PDE3, and PDE4. PDE1 and PDE5 are mainly responsible for cGMP-hydrolyzing activity, whereas PDE3 and PDE4 contribute to most of cAMP-hydrolyzing activity $[15,16]$. PDE4 has four subfamilies: PDE4A, 4B, 4C, and 4D, which are differentially localized between cells [17]. PDE4D is expressed broadly in many kinds of cell $[18,19]$. In arterial VSMCs, PDE4D is dominantly expressed and degrades second-messenger cAMP[20]. Recent studies suggest that PDE4D may have a critical role in atherosclerosis. For example, PDE4D is largely associated with atherosclerotic stroke such as cardiogenic and carotid strokes [1]. Furthermore, a decrease in cAMP level has been found to associate with increased proliferation and migration of vascular smoothmuscle cells, the central events in the development of atherosclerosis $[21,22]$. More importantly, PDE4 antagonists have been shown to significantly inhibit smooth-muscle proliferation in a rat carotid balloon-injury model [23-25]. However, the reagents they used are not the specific inhibitor against PDE4D, which makes it hard to determinate the functional roles of PDE4D isoform in the process. Therefore, 
genetic approach specifically targeting PDE4D gene such as use of shRNA to knockdown its expression would be necessary to address this issue.

The present study was conducted to investigate whether downregulation of PDE4D can inhibit VSMC proliferation and migration. Lentivirus particle carrying small hairpin RNA against PDE4D was applied to specifically reduce PDE4D expression in rat aortic smooth muscle cells. We found that PDE4D silence in rat aortic smooth muscle cells significantly inhibits their proliferation and migration induced by platelet-derived growth factor (PDGF), and the inhibition is not associated with global intracellular cAMP level.

\section{Materials and Methods}

2.1. Cell Culture. The human HEK-293T-cell line was obtained from American Type Culture Collection (ATCC) and grown in RPMI 1640 supplemented with 10\% FBS (Sigma-Aldrich, St. Louis, MO, USA).

VSMCs were isolated from descending thoracic aorta of male Sprague-Dawley rats weighed about $150 \mathrm{~g}$ and characterized morphologically and immunohistochemically as described previously [26]. Cells were grown in Dulbecco's modified Eagle's medium (DMEM), with 10\% fetal bovine serum (FBS), $1 \mathrm{mmol} / \mathrm{L}$ L-glutamine, $100 \mathrm{U} / \mathrm{mL}$ penicillin, and $100 \mu \mathrm{g} / \mathrm{mL}$ streptomycin, at $37^{\circ} \mathrm{C} / 5 \% \mathrm{CO}_{2}$. Cells were trypsin passaged after 10 days and then at $95 \%$ confluence (Jinuo Biology and Medicine Company, China). All experiments were performed on cells at passage 4 through 6 and $60 \%$ to $90 \%$ confluence. In all experiments, the cells were made quiescent by incubating for 24 hours in serum-free medium prior to use.

2.2. PDE4D Gene Silence in Rat VMSCs. The lentivirus vector system used in the experiment was purchased from Shanghai Genchem Company (Shanghai, China), which was composed of three plasmids-pGCL-GFP, pHelper1.0, and pHelper2.0. The plasmid pGCL-GFP had an U6 promoter and a GFP reporter gene.

The oligonucleotide sequences of four different PDE4DshRNAs and a control nonsense shRNA are as shown in Table 1. All shRNAs were synthesized by Shanghai Genchem Company. These four 21 nucleotide shRNA duplexes from four different parts of the PDE4D mRNA (Gen Bank Accession no. NM_017032) were designed using specific guidelines for designing shRNA hairpins encoded by shRNA expression vectors and shRNA expression cassettes of Ambion company. Of sequences tested for PDE4D knockdown using Western blot, the sequence locating at 181 achieved the best interfering effect and was subsequently cloned into the shRNApGCL-GFP lentiviral vector. Nonsense shRNA was served as a control. Infectious viruses were produced by cotransfecting the lentiviral vectors and packaging constructs into 293FT cells using Lipofectamine reagent (Invitrogen).

Recombinant lentviruses containing shRNA against PDE4D and nonsense shRNA were obtained from Genchem Company. VSMCs were infected with purified lentivirus at
TABLE 1: Information of four designed shRNA and nonsense sequences.

\begin{tabular}{lccc}
\hline No. & Sequences & Locates & GC $\%$ \\
\hline 1 & GCGATTATGACCTCTCTCC & 181 & 52.60 \\
2 & CCAACCATCCATCAACAAA & 368 & 42.10 \\
3 & CCCATGTGCAACCAACCAT & 357 & 52.60 \\
4 & CCGGATAATGGAGGAGTTC & 1499 & 52.60 \\
nonsense & TTCTCCGAACGTGTCACGT & - & 52.63 \\
\hline
\end{tabular}

The no. 1 locating at 181 achieved the best interfering effect around $72 \%$.

an MOI (multiplicities of infection) of 50 to obtain $95-100 \%$ efficiency, as determined by GFP expression 24 hours after infection.

2.3. Western Blot. VSMCs were collected and sonicated by sonicator. Protein was extracted from the lysate and separated with SDS-PAGE (sodium dodecyl sulfate polyacrylamide gel electrohoresis). Following electrophoresis, proteins were transferred to PVDF membranes, and the membranes were blocked by incubation with TBST (Tris Buffered Saline Tween) containing 5\% skim milk for 1 hour. Blots were incubated with an appropriate dilution of primary antibodies against rat PDE4D (Abcam) and $\alpha$-actin (Santacruz) for 2 hours and rinsed three times with TBST. Rinsed blots were incubated with second antibodies (Santacruz) for 2 hours and rinsed with TBST. Immunoreactivity was detected by chemiluminescence (Amersham ECL plus kit).

2.4. Cell Viability and Proliferation. Cell viability and proliferation was measured by assessing mitochondrial activity of VSMCs using an MTT kit (Sigma). Cells were growtharrested in serum-free medium for 24 hours and then cultured in medium with $0.5 \%$ FBS and PDGF-BB (Pepro Tech, $20 \mathrm{ng} / \mathrm{mL})$ for 24 hours before addition of MTT $(5 \mathrm{mg} / \mathrm{mL}$, Boli Company, China). The MTT solution $(5 \mathrm{mg} / \mathrm{mL}$ MTT in medium without phenol red) was added to the culture solution to a volume equal to $10 \%$. Further incubation at $37^{\circ} \mathrm{C}$ for four hours yielded purple MTT formazan crystals. Once solubilized with DMSO, the absorbance in each culture was measured at $570 \mathrm{~nm}$ with the background $(650 \mathrm{~nm})$ subtracted.

2.5. Migration Assay. VSMCs migration assays were performed using a modified Boyden's chamber fitted with $8-\mu \mathrm{m}$ pore membrane (Corning). Briefly, VSMCs were resuspended in serum-free medium to a concentration of $1 \times 10^{5}$ cells $/ \mathrm{mL}$. A volume of $200 \mu \mathrm{L}$ of cell suspension was added to the upper wells of the Boyden's chamber, and 0.5\% FBS-medium with or without PDGF $(20 \mathrm{ng} / \mathrm{mL})$ was added to the lower chamber. Migration was allowed to proceed for 12 hours in a $37^{\circ} \mathrm{C}, 5 \% \mathrm{CO}_{2}$, humidified atmosphere. Then, cells remaining on the upper surface of the membrane were scraped off with cotton sticks. Cells that had migrated to the lower surface were fixed with paraformaldehyde (Guangzhou Reagent factory) and stained with DAPI (Jackson) and crystal violet (Weijia Technical Company, Guangzhou). Migration 


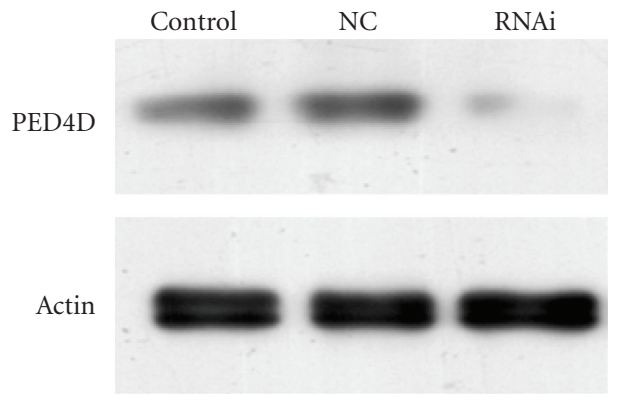

(a)

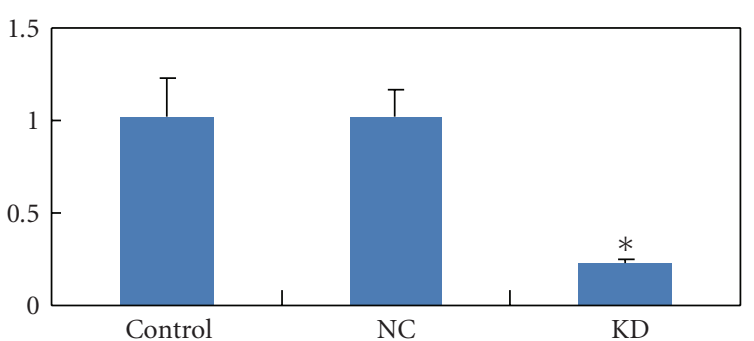

(b)

FIGURE 1: Knockdown of endogenous PDE4D expression by lentiviral vector-mediated shRNA in VSMCs. VSMCs were infected with the same amount (PDE4D-shRNA: $12.5 \mu \mathrm{L}$, nonsense shRNA: $6.25 \mu \mathrm{L}$ ) of lentivirus particles carrying either shRNA (titer $4 \times 10^{8} \mathrm{TU} / \mathrm{mL}$ ) against PDE4D or nonsense shRNA (titer $8 \times 10^{8} \mathrm{TU} / \mathrm{mL}$ ). PDE4D protein expression was determined at 48 hours postinfection. Values represent means \pm SEM. E: inhibition of PDE4D expression in PDE4D shRNA-tranfected cells was calculated relative to the PDE4D expression in cells tranfected with the control nonsense shRNA. Sh-GFP: lentivirus particles carrying nonsense shRNA, Sh-PDE4D: lentivirus particles carrying shRNA against PDE4D.

capacity was identified by the average number of stained cells in six random fields of view (magnification $\times 400$ ) per membrane. The experiment was repeated three times.

2.6. Measurement of $c A M P$. The intracellular cAMP levels in cultured VSMCs were determined by cAMP RIA test kit purchased from Shanghai Chinese Medicine University following the kit instruction (Shanghai, China). The detection limit of cAMP was $0.08 \mathrm{pmol} / \mathrm{L}$, and the interand intra-assay coefficients of variation were $<10 \%$. Starved cells were incubated in $0.5 \%$ FBS-medium with or without PDGF $(20 \mathrm{ng} / \mathrm{mL})$ for another 24 hours. Cells were lysed in acid/alcohol $(750 \mathrm{~mL}$ of ethanol: $248.5 \mathrm{~mL}$ of doubledistilled $\mathrm{H}_{2} \mathrm{O}: 1.5 \mathrm{~mL}$ of concentrated $\mathrm{HCL}$ ) containing IBMX $(500 \mu \mathrm{M})$. The lysate was acetylated with $10 \mu \mathrm{L}$ volume of a $1: 2(\mathrm{v}: \mathrm{v})$ mixture of acetic anhydride plus triethylamine. After acetylation, samples were preincubated for 1 hour at room temperature with $100 \mu \mathrm{L}$ anti-cAMP before adding $100 \mu \mathrm{L}{ }^{125} \mathrm{I}$-cAMP and incubating for overnight at $4^{\circ} \mathrm{C}$. After separation with adsorbing materials, the samples were centrifuged at $3000 \mathrm{rpm}$ for $15 \mathrm{~min}$. The supernatant fluids were aspirated, the radioactivity of precipitates was counted by $\gamma$-counter, and cAMP levels were determined by comparison to a standard curve.

2.7. Statistical Analysis. All data were processed with SPSS 12.0 statistical software. All results are expressed as means \pm SEM. Data were analyzed using a one-way ANOVA and Student-Newman-Keuls tests for multiple comparisons. In all cases, $P \leq .05$ was taken as statistically significant.

\section{Results}

3.1. Screening of shRNAs for Gene Silence of PDE4D after Infection with Lentivrius Particles Carrying shRNA against PDE4D in VSMCs. To determine PDE4D gene silencing effect, VSMCs were transiently transfected with lentivrius particles carrying shRNAs against PDE4D. Four different
shRNA duplexes, named as no. 1, no. 2, no. 3, and no. 4 , were designed to target different regions of rat PDE4D (NM_017032): start sites of 181, 368, 357, and 1499 (Table 1). All four different shRNAs could inhibit PDE4D at both transcription and protein levels as determined by real-time RTPCR and western blotting (data not shown). These shRNA duplexes showed varying degrees of PDE4D gene silencing efficiency ranging from $53 \%$ to $72 \%$ (data not shown). The no. 1 with sequence locating at 181 achieved the best interfering effect around $72 \%$ and was subsequently cloned into the shRNA-pGCL-GFP lentiviral vector (Figure 1). The nonsense shRNA treatment did not significantly alter the levels of PDE4D transcript and protein and was subsequently cloned into the shRNA- pGCL-GFP lentiviral vector as a shRNA control (Figure 1).

3.2. PDE4D Gene Silence Inhibits PDGF-Induced VSMC Proliferation without Affecting Cell Viability. To determine whether PDE4D participates in PDGF-induced VSMC proliferation, we treated cells with PDGF and the proliferation was assayed using MTT at 24 hours after PDGF stimulation. As shown in Figure 2, noninfected control cells and cells infected with lentivirus particles carrying either shRNA direct against PDE4D or nonsense shRNA grew at similar rates. Cells treated with PDGF were increased by almost threefolds when compared to untreated cells. Infection of VSMCs with lentivius particles carrying shRNA direct against PDE4D significantly resulted in about $30 \%$ reduction of PDGF-induced proliferation. In contrast, infection of VSMCs with the lentivirus particle carrying nonsense shRNA did not significantly affect PDGF-induced proliferation. This result demonstrates an important role of PDE4D in PDGF directed VSMC proliferation.

3.3. PDE4D Gene Silence Suppresses Migration. To determine whether PDE4D gene contributes to VSMC migration, we silenced gene expression using lentivirus particle carrying 


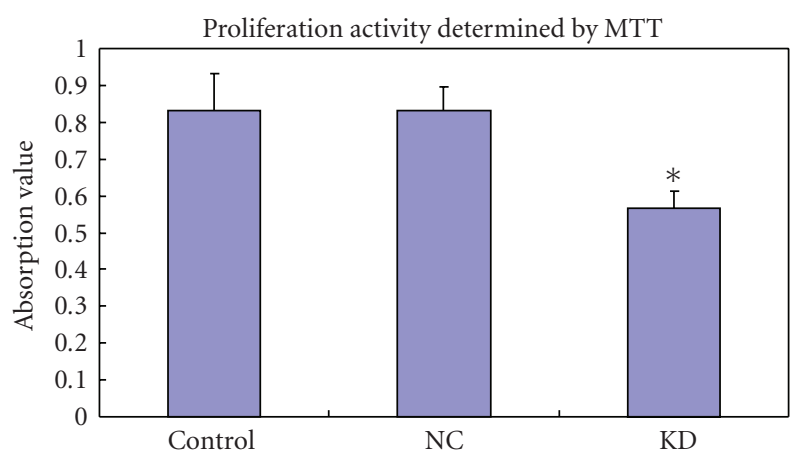

FIGURE 2: PDE4D regulates PDGF-induced VSMC proliferation. VSMC viability was determined using MTT assay at 48 hours postinfection. Data are mean \pm SEM of 3 separate experiments performed in triplicate. ${ }^{*} P$ less than .05 versus control. ${ }^{*} P$ less than .05 versus nonsense shRNA.

shRNA against PDE4D and evaluated migration using Boyden's chamber. Rat aortic VSMCs were made quiescent by 24 hour treatment in serum-free culture medium. As shown in Figure 3, noninfected control cells and cells infected with lentivirus encoding either shRNA direct against PDE4D or nonsense shRNA had similar migration rates. Treatment with PDGF significantly increased VSMC migration up to sixfolds when compared to untreated cells. Infection of VSMCs with lentivirus particle carrying shRNA direct against PDE4D significantly resulted in almost 39\% reduction of PDGF-induced migration. In contrast, infection of VSMCs with the lentivirus carrying nonsense shRNA did not significantly affect PDGF-induced migration.

3.4. Intracellular cAMP Level. To examine whether the inhibitory effects of PDE4D gene silence on VSMC proliferation and migration were mediated through intracellular cAMP, we measured cAMP levels by enzyme immunoassay after infection of VSMCs with lentivrus carrying shRNA against PDE4D gene (Figure 4). Infection of VSMCs with lentivrus particle carrying either shRNA against PDE4D gene or nonsense shRNA did not result in any significant change in VSMC basal level of cAMP. The addition of PDGF $(20 \mathrm{ng} / \mathrm{mL})$ stimulated a statistically significant accumulation of cAMP in VSMCs compared with control. However, infection of VSMCs with lentivirus particle carrying shRNA against PDE4D gene or nonsense shRNA did not result in any significant change in PDGF-stimulated accumulation of cAMP.

\section{Discussion}

In the present study, we studied the functional consequence of PDE4D knockdown by RNA interference in PDGFinduced VSMC proliferation and migration. We found that knockdown of endogenous PDE4D significantly reduces rat aortic VSMC proliferation and migration. However, the inhibition does not lead to alteration of the global intracellular cAMP level, suggesting that the inhibition is less likely mediated through cAMP.
Atherosclerosis is a major cause of ischemic stroke [27]. Vascular endothelial cell injury and subsequent migration and proliferation of VSMCs are early key events of atherosclerosis. We here used cultured VSMCs after three passages as a model to study VSMC proliferation and migration because they represent VSMCs in a synthetic phenotype which is similar to migrating smooth muscle cells in vivo. Platelet-derived growth factor (PDGF), one of the most potent mitogens and chemoattractants for VSMCs produced by platelets, VSMCs, and endothelial cells in the injured vascular wall, plays the central role in the onset and development of vascular disorders $[28,29]$. Therefore, VSMC proliferation and migration in this study were induced by the addition of the potent and pathophysiologically relevant chemotactic factor PDGF. As shown in Figure 2, we confirmed that PDGF caused proliferation in rat VSMCs. In addition, we observed about 30\% reduction of PDGF-induced cell proliferation in PDE4D deficient VSMCs. We believe that the reduction of PDGF-induced cell proliferation in PDE4D deficient VSMCs is specific because knockdown of PDE4D had no effect on quiescent VSMCs where PDGF was absent. This finding is consistent with previous studies that Rolipram, a general PDE4 inhibitor, can inhibit proliferation of VSMCs from $26 \%$ to $37 \pm 6 \%$ [30-32]. It is not surprised to note that knockdown of PDE4D did not achieve a full suppression of VSMC proliferation because multiple signals are involved in PDGF-induced VSMC proliferation. Furthermore, the members of PDE family in addition to PDE4D have been shown to closely associate with VSMC proliferation. For example, inhibition of PDE3 and PDE1A also reduce VSMC proliferation $[33,34]$. Nevertheless, our data suggest that PDE4D is important in PDGF-induced VSMC proliferation.

Although VSMC migration plays a pivotal role in the pathogenesis of arteriosclerosis, the role of PDE4D in VSMC migration has received much less attention. Few investigators have studied the effects of PDE4D on VSMC migration. The results are inconsistent in those limited reports. For example, Goncharova and his colleagues found that cilomolast, a PDE4 inhibitor can inhibit basal (unstimulated) and PDGFstimulated migration of airway smooth muscle and pulmonary vascular smooth muscle cells [35]. Palmer and his colleagues reported that Ro 20-1724, another PDE4 inhibitor with potent effect on PDE4D, potentiated antimigratory effect caused by forskolin [25]. However, neither cilomolast nor Ro 20-1724 is the specific inhibitor against PDE4D. As a result, lack of selective inhibitors against PDE4D makes it difficult to determinate the functional roles of PDE4D isoform. To overcome this problem, we used shRNA techniques to selectively knockdown PDE4D expression in VMSCs. We found that knockdown of PDE4D significantly inhibited PDGF-induced migration. This effect is in parallel to the inhibitory action of PDE4D on VMSC proliferation.

It has been well documented that cAMP and its signaling play a critical role in regulating VSMC proliferation and migration $[21,22,36]$. In addition, cAMP is the only substrate of PDE4D. Therefore, we hypothesized that the inhibitory effects of knockdown of PDE4D might be mediated through its regulation of cAMP. Although PDGFinduced robust production of cAMP in VMSC cells, we failed 


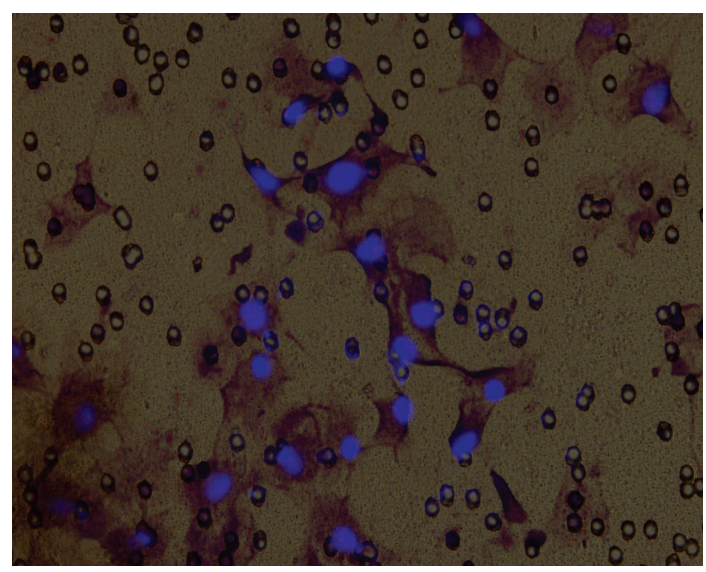

(a)

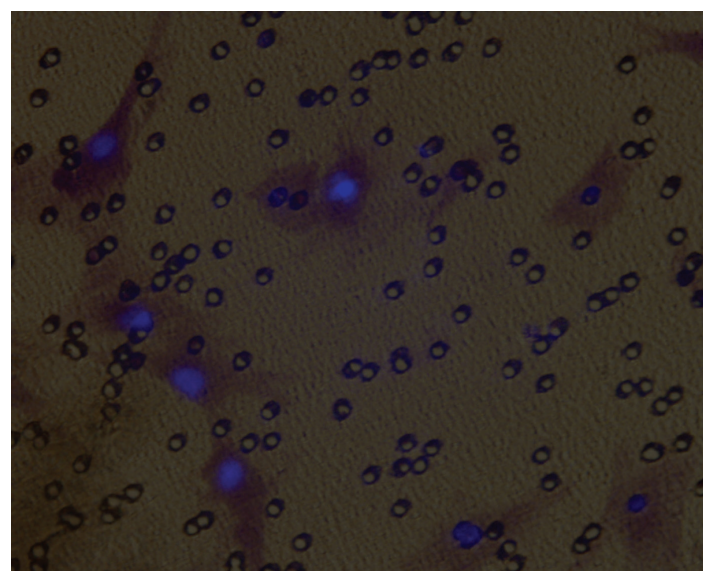

(c)

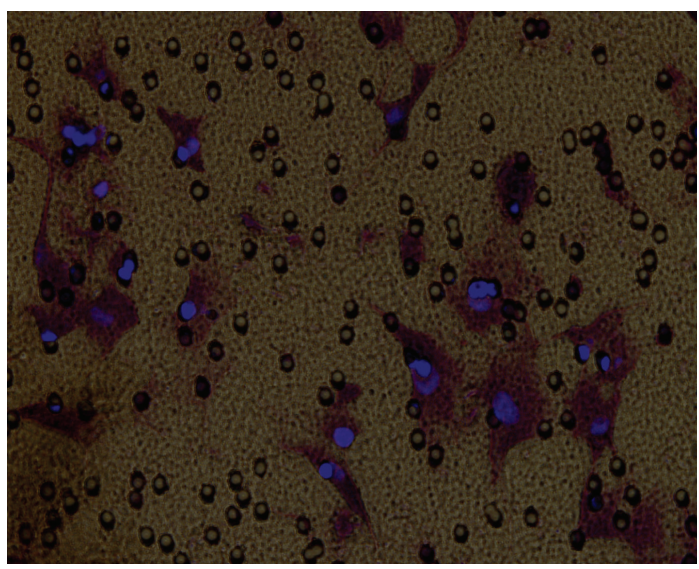

(b)

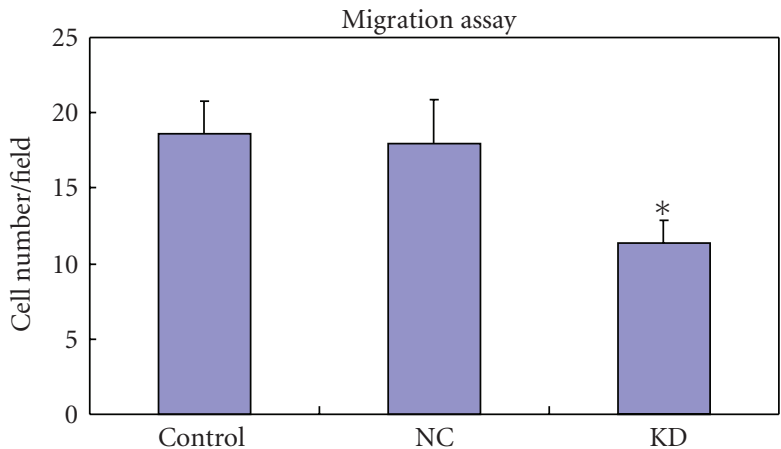

(d)

FIGURE 3: Knockdown of endogenous PDE4D inhibits PDGF-induced VSMC migration. PDGF-induced VSMC migration was assessed in a Boyden's chamber. Representative images ( $, b, c)$ and group data (d) are shown. (a)-(c) Representative photomicrographs illustrating migration of VSMCs. (a) Migration of VSMCs stimulated by PDGF-BB. (b) Migration of VSMCs stimulated by PDGF-BB in cells infected with lentivirus particles carrying nonsense shRNA. (c) Migration of VSMCs stimulated by PDGF-BB in cells infected with lentivrius carrying shRNA against PDE4D. (d) Bar graph illustrating PDGF-BB-induced VSMC migration in noninfected cells and cells infected with lentivirus particles carrying either PDE4D or nonsense shRNA. Numbers of migrated VSMCs were much less in cells infected with lentivrius carrying PDE4D shRNA compared with noninfected cells or cells infected with lentivirus particles carrying nonsense shRNA. Bars are the mean plus minus SEM of six random fields of view from three separate experiments. Original magnification, $\times 400 .{ }^{*} P$ less than .05 versus control. ${ }^{\#} P$ less than .05 versus nonsense shRNA.

to detect any significant change of cAMP level in PDGFinduced PDE4D deficient VMSC which is consistent with several previous reports $[28,29]$. Given that intracellular cAMP level is modulated by PDE family members, it is reasonable that inhibition of PDE4 or PDE4D alone may not be able to affect the global level of cAMP in cells. The other possible explanation is that the effect of PDE4 family on VSMC proliferation and migration may not be entirely mediated by change in intracellular cAMP concentration. In addition to its action on cAMP-signaling, PDE4D also has a cross-talk with extracellular-signal-regulated kinases (ERK) in response to both cAMP-dependent and non-cAMPdependent agents. ERK has been reported to be important for PDGF- stimulated proliferation and migration of VSMC.
In addition, cAMP are distributed with spatial gradient, not freely $[37,38]$. Therefore, cAMP induced by PDE4 or other family member can be highly compartmentalized and insufficient to increase total cellular cAMP. This may also explain the failure to detect a significant change in cAMP after single inhibition of PDE4. Therefore, we speculate that knockdown of PDE4D may alter local concentration of cAMP and thus regulate its signaling locally to inhibit proliferation and migration of cells.

\section{Conclusion}

In this study, we infect VSMC with lentivrius particles carrying shRNA direct against PDE4D. Consequently, VSMC 


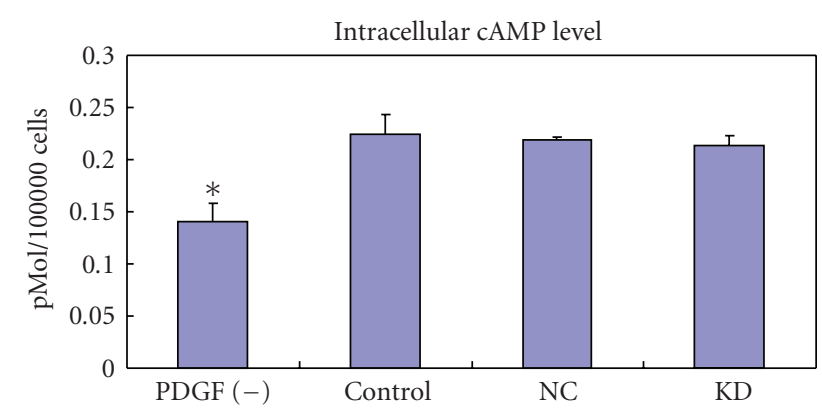

Figure 4: Knockdown of endogenous PDE4D does not affect PDGF-induced cAMP level in VSMC. Bar graph illustrates PDGFBB induced cAMP level in noninfected cells and cells infected with lentivirus particles carrying either PDE4D or nonsense shRNA. There were no significant differences in cAMP level between cells infected with lentivrius carrying PDE4D shRNA and noninfected cells, cells infected with lentivirus particles carrying nonsense shRNA. Values represent means \pm SEM from three separate experiments. ${ }^{*} P$ less than .05 versus control.

proliferation and migration was significantly inhibited, and the inhibitory effects were not associated with global intracellular cAMP level. Our results implicate that PDE4D has an important role in VSMC proliferation and migration which may explain its genetic susceptibility to ischemic stroke.

\section{Acknowledgment}

The authors would like to offer special thanks to Dr. Zhong Pei for manuscript preparation. This study was supported by Grants from the National Natural Science Foundation of China (no. 39700048, 302713781, 81070912/H0905), the Natural Science Foundation of Guangdong Province (no. 06021225, 021866, 980066, 974151), the Key Project of Guangdong Provincial Committee on Science and Technology (nos. 2005B31201013, 2002B50301009, 2002C30603), and the Special Research Foundation for Doctoral Disciplines of Colleges and Universities (no. 20070558234). The funding source was not involved in study design; collection, analysis, and interpretation of data; writing of the report; or decision to submit the paper for publication.

\section{References}

[1] S. Gretarsdottir, G. Thorleifsson, S. Th. Reynisdottir et al., "The gene encoding phosphodiesterase $4 \mathrm{D}$ confers risk of ischemic stroke," Nature Genetics, vol. 35, no. 2, pp. 131-138, 2003.

[2] N. Li, Z. He, J. Xu, F. Liu, S. Deng, and H. Zhang, "Association of PDE4D and IL-1 gene polymorphism with ischemic stroke in a Han Chinese population," Brain Research Bulletin, vol. 81, no. 1, pp. 38-42, 2010.

[3] A. Munshi, M. S. Babu, S. Kaul et al., "Phosphodiesterase 4D (PDE4D) gene variants and the risk of ischemic stroke in a South Indian population," Journal of the Neurological Sciences, vol. 285, no. 1-2, pp. 142-145, 2009.

[4] T. Matsushita, M. Kubo, K. Yonemoto et al., "Lack of association between variations of PDE4D and ischemic stroke in the Japanese population," Stroke, vol. 40, no. 4, pp. 12451251, 2009.

[5] K. Kostulas, S. Gretarsdottir, V. Kostulas et al., "PDE4D and ALOX5AP genetic variants and risk for Ischemic Cerebrovascular Disease in Sweden," Journal of the Neurological Sciences, vol. 263, no. 1-2, pp. 113-117, 2007.

[6] Q. Song, J. W. Cole, J. R. O’Connell et al., "Phosphodiesterase 4D polymorphisms and the risk of cerebral infarction in a biracial population: the Stroke Prevention in Young Women Study," Human Molecular Genetics, vol. 15, no. 16, pp. 24682478, 2006.

[7] D. Woo, R. Kaushal, B. Kissela et al., "Association of phosphodiesterase 4D with ischemic stroke: a population-based casecontrol study," Stroke, vol. 37, no. 2, pp. 371-376, 2006.

[8] E. Lohmussaar, A. Gschwendtner, J. C. Mueller et al., "ALOX5AP gene and the PDE4D gene in a central European population of stroke patients," Stroke, vol. 36, no. 4, pp. 731736, 2005.

[9] S. Bevan, L. Porteous, M. Sitzer, and H. S. Markus, "Phosphodiesterase 4D gene, ischemic stroke, and asymptomatic carotid atherosclerosis," Stroke, vol. 36, no. 5, pp. 949-953, 2005.

[10] X. Xu, X. Li, J. Li, R. Ou, and W. Sheng, "Meta-analysis of association between variation in the PDE4D gene and ischemic cerebral infarction risk in Asian populations," Neurogenetics, vol. 11, no. 3, pp. 327-333, 2010.

[11] P. J. Newcombe, C. Verzilli, J. P. Casas, A. D. Hingorani, L. Smeeth, and J. C. Whittaker, "Multilocus Bayesian metaanalysis of gene-disease associations," American Journal of Human Genetics, vol. 84, no. 5, pp. 567-580, 2009.

[12] S. Bevan, M. Dichgans, A. Gschwendtner, G. Kuhlenbaumer, E. B. Ringelstein, and H. S. Markus, "Variation in the PDE4D gene and ischemic stroke risk: a systematic review and metaanalysis on 5200 cases and 6600 controls," Stroke, vol. 39, no. 7, pp. 1966-1971, 2008.

[13] J. M. Staton, M. S. Sayer, G. J. Hankey et al., "Association between phosphodiesterase 4D gene and ischaemic stroke," Journal of Neurology, Neurosurgery and Psychiatry, vol. 77, no. 9, pp. 1067-1069, 2006.

[14] D. M. Essayan, "Cyclic nucleotide phosphodiesterases," Journal of Allergy and Clinical Immunology, vol. 108, no. 5, pp. 671-680, 2001.

[15] D. Kim, S. D. Rybalkin, X. Pi et al., "Upregulation of phosphodiesterase 1A1 expression is associated with the development of nitrate tolerance," Circulation, vol. 104, no. 19, pp. 23382343, 2001.

[16] D. Kim, T. Aizawa, H. Wei et al., "Angiotensin II increases phosphodiesterase 5A expression in vascular smooth muscle cells: a mechanism by which angiotensin II antagonizes cGMP signaling," Journal of Molecular and Cellular Cardiology, vol. 38, no. 1, pp. 175-184, 2005.

[17] T. Muller, P. Engels, and J. R. Fozard, "Subtypes of the type 4 cAMP phosphodiesterases: structure, regulation and selective inhibition," Trends in Pharmacological Sciences, vol. 17, no. 8, pp. 294-298, 1996.

[18] G. Némoz, R. Zhang, C. Sette, and M. Conti, "Identification of cyclic AMP-phosphodiesterase variants from the PDE4D gene expressed in human peripheral mononuclear cells," FEBS Letters, vol. 384, no. 1, pp. 97-102, 1996.

[19] D. Peter, S. L. C. Jin, M. Conti, A. Hatzelmann, and C. Zitt, "Differential expression and function of phosphodiesterase 4 (PDE4) subtypes in human primary CD4+ T cells: predominant role of PDE4D," Journal of Immunology, vol. 178, no. 8, pp. 4820-4831, 2007. 
[20] H. Liu and D. H. Maurice, "Phosphorylation-mediated activation and translocation of the cyclic AMP- specific phosphodiesterase PDE4D3 by cyclic AMP-dependent protein kinase and mitogen-activated protein kinases: a potential mechanism allowing for the coordinated regulation of PDE4D activity and targeting," Journal of Biological Chemistry, vol. 274, no. 15, pp. 10557-10565, 1999.

[21] X. J. Cai, "Regulation of smooth muscle cells in development and vascular disease: current therapeutic strategies," Expert Review of Cardiovascular Therapy, vol. 4, no. 6, pp. 789-800, 2006.

[22] H. Koyama, K. E. Bornfeldt, S. Fukumoto, and Y. Nishizawa, "Molecular pathways of cyclic nucleotide-induced inhibition of arterial smooth muscle cell proliferation," Journal of Cellular Physiology, vol. 186, no. 1, pp. 1-10, 2001.

[23] C. Indolfi, E. V. Avvedimento, E. Di Lorenzo et al., "Activation of cAMP-PKA signaling in vivo inhibits smooth muscle cell proliferation induced by vascular injury," Nature Medicine, vol. 3, no. 7, pp. 775-779, 1997.

[24] C. Indolfi, E. Di Lorenzo et al., "8-chloro-cAMP inhibits smooth muscle cell proliferation in vitro and neointima formation induced by balloon injury in vivo," Journal of the American College of Cardiology, vol. 36, no. 1, pp. 288-293, 2000.

[25] D. Palmer, K. Tsoi, and D. H. Maurice, "Synergistic inhibition of vascular smooth muscle cell migration by phosphodiesterase 3 and phosphodiesterase 4 inhibitors," Circulation Research, vol. 82, no. 8, pp. 852-861, 1998.

[26] J. Chamley-Campbell, G. R. Campbell, and R. Ross, "The smooth muscle cell in culture," Physiological Reviews, vol. 59, no. 1, pp. 1-61, 1979.

[27] G. K. Owens, M. S. Kumar, and B. R. Wamhoff, "Molecular regulation of vascular smooth muscle cell differentiation in development and disease," Physiological Reviews, vol. 84, no. 3, pp. 767-801, 2004.

[28] R. Ross, "Platelet-derived growth factor," Lancet, vol. 1, no. 8648, pp. 1179-1182, 1989.

[29] R. Ross, "The pathogenesis of atherosclerosis: a perspective for the 1990s," Nature, vol. 362, no. 6423, pp. 801-809, 1993.

[30] J. E. Souness, G. A. Hassall, and D. P. Parrott, "Inhibition of pig aortic smooth muscle cell DNA synthesis by selective type III and type IV cyclic amp phosphodiesterase inhibitors," Biochemical Pharmacology, vol. 44, no. 5, pp. 857-866, 1992.

[31] K. Johnson-Mills, E. Arauz, R. G. Coffey, J. J. Krzanowski, and J. B. Polson, "Effect of CI-930 [3-(2H)-pyridazinone4,5-dihydro-6-[4-(1H-imidazolyl) phenyl]-5-methylmonohydrochloride] and rolipram on human coronary artery smooth muscle cell proliferation," Biochemical Pharmacology, vol. 56, no. 8, pp. 1065-1073, 1998.

[32] E. J. Growcott, K. G. Spink, X. Ren, S. Afzal, K. H. Banner, and J. Wharton, "Phosphodiesterase type 4 expression and anti-proliferative effects in human pulmonary artery smooth muscle cells," Respiratory Research, vol. 7, article 9, 2006.

[33] Y. Inoue, K. Toga, T. Sudo et al., "Suppression of arterial intimal hyperplasia by cilostamide, a cyclic nucleotide phosphodiesterase 3 inhibitor, in a rat balloon double-injury model," British Journal of Pharmacology, vol. 130, no. 2, pp. 231-241, 2000.

[34] D. J. Nagel, T. Aizawa, K.-I. Jeon et al., "Role of nuclear $\mathrm{Ca} 2+/$ calmodulin-stimulated phosphodiesterase $1 \mathrm{~A}$ in vascular smooth muscle cell growth and survival," Circulation Research, vol. 98, no. 6, pp. 777-784, 2006.

[35] E. A. Goncharova, C. K. Billington, C. Irani et al., "Cyclic AMP-mobilizing agents and glucocorticoids modulate human smooth muscle cell migration," American Journal of Respiratory Cell and Molecular Biology, vol. 29, no. 1, pp. 19-27, 2003.

[36] H. Ono, T. Ichiki, K. Fukuyama et al., "cAMP-response element-binding protein mediates tumor necrosis factoralpha-induced vascular smooth muscle cell migration," Arteriosclerosis, Thrombosis, and Vascular Biology, vol. 24, no. 9, pp. 1634-1639, 2004.

[37] J. Zhang, Y. Ma, S. S. Taylor, and R. Y. Tsien, "Genetically encoded reporters of protein kinase A activity reveal impact of substrate tethering," Proceedings of the National Academy of Sciences of the United States of America, vol. 98, no. 26, pp. 14997-15002, 2001.

[38] M. Mongillo, T. McSorley, S. Evellin et al., "Fluorescence resonance energy transfer-based analysis of cAMP dynamics in live neonatal rat cardiac myocytes reveals distinct functions of compartmentalized phosphodiesterases," Circulation Research, vol. 95, no. 1, pp. 67-75, 2004. 


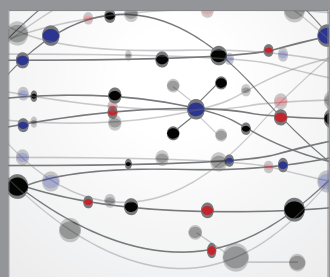

The Scientific World Journal
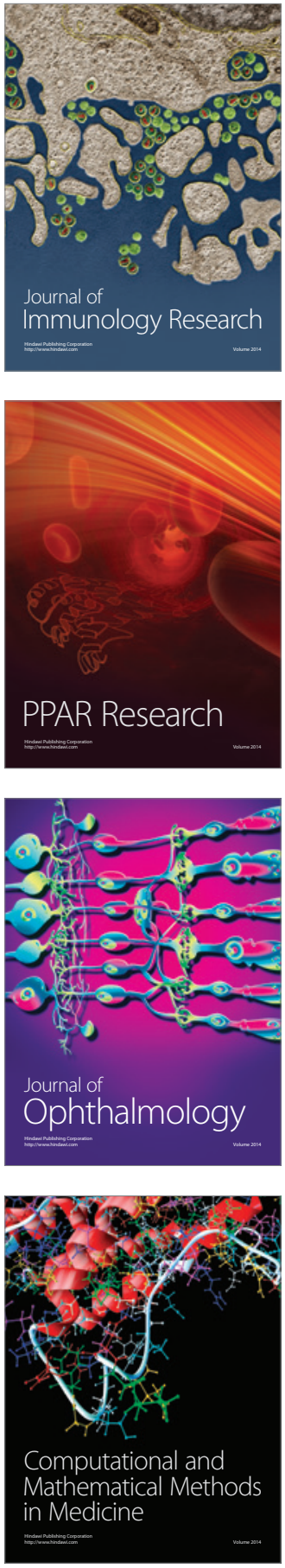

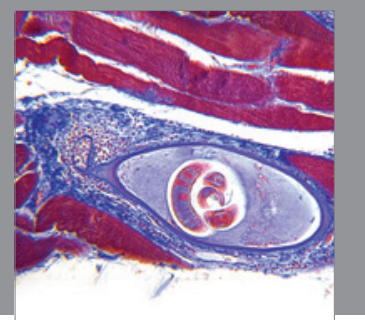

Gastroenterology

Research and Practice
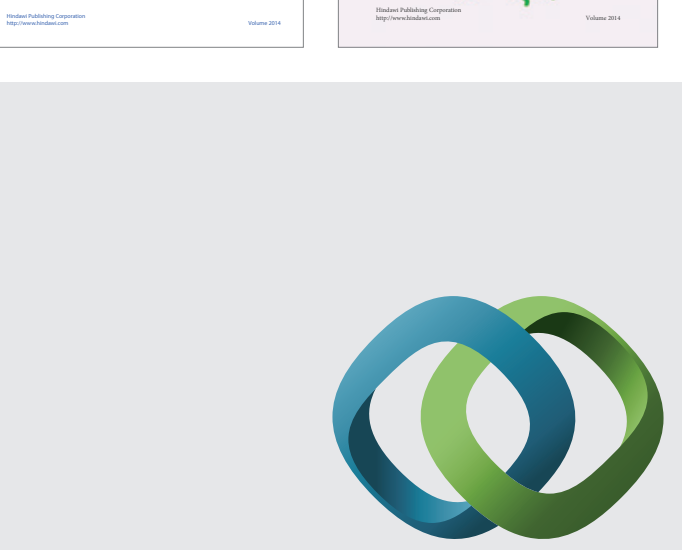

\section{Hindawi}

Submit your manuscripts at

http://www.hindawi.com
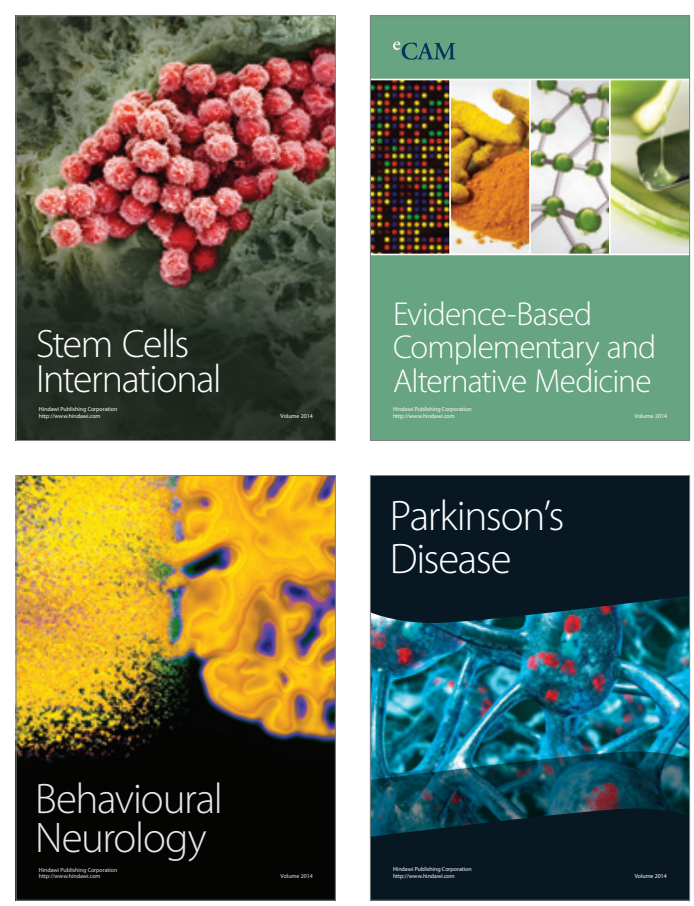

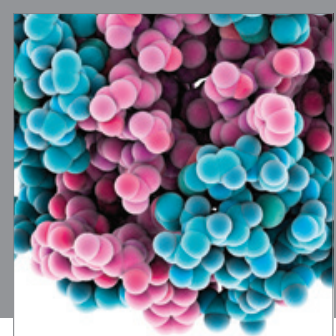

Journal of
Diabetes Research

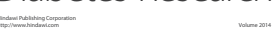

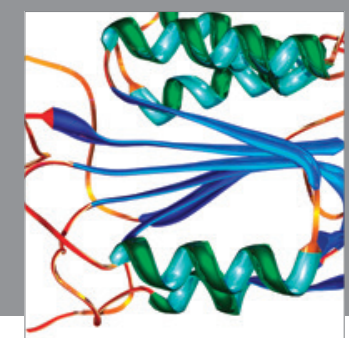

Disease Markers
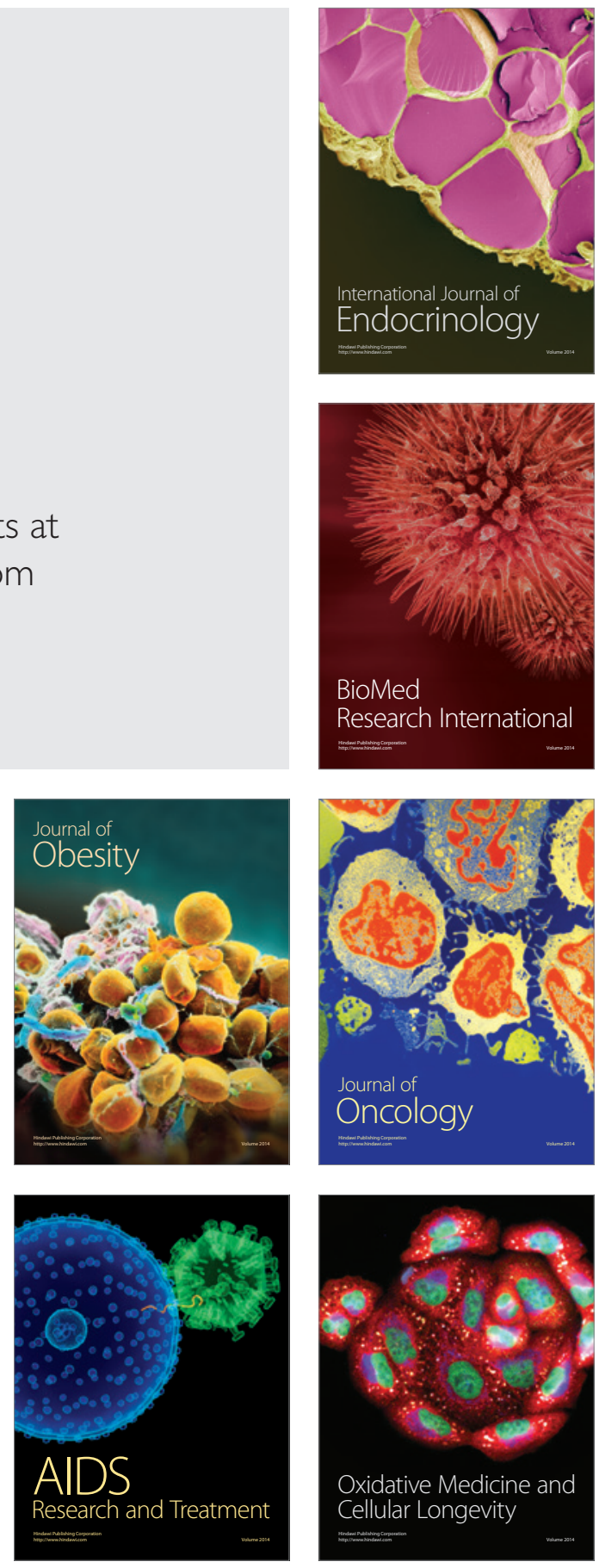